\title{
OLTRE IL TESTO: LO SPECIFICO FEMMINILE NELL'EDITORIA
}

\author{
BEYOND THE TEXT: WOMEN IN PUBLISHING FIELD
}

Luca De Feo

\section{RIASSUNTO:}

Questo articolo è uno dei primi che parladi come le donne editano. La sensibilità femminile può modificare l'approccio al prodotto e alla vendita. Oggi il merito delle donne nell'editoria è riconosciuto e nelle piccole case editrici la metà dei dirigenti sono donne. Verrà analizzato il ruolo delle donne nell'editoria nel secolo scorso ma soprattutto si concentra sulle nuove tendenze: siamo nell'epoca degli e-book.

\section{PAROLE CHIAVE:}

Donne, case editrici, e-book, giornaliste.

\section{ABSTRACT:}

This article is one of the first about how women publish. Their sensibility can influence products and marketing. Nowadays the merit of women in publishing is recognized, and half of the directors of small publishing houses are women. It is analyzed the role of women during last century but the focus is put on new trends: we are living the e-book age.

\section{KEY wORD:}

Women, publishing houses, e-book, women journalists. 


\section{UN APPROCCIO DIVERSO: TESTO E CONTESTO}

Nel 1965 tra gli Oscar Mondadori uscirono i Malavoglia. Il formato era tascabile, sulla copertina, a colori, Padron'Toni aveva fattezze quasi cinematografiche. Fino ad allora il romanzo di Verga era stato un classico da liceo; Mondadori lo rendeva una saga da sfogliare sul tram o sotto l'ombrellone. Effetti, in fondo, di ciò che Gerard Genette chiama peritesto e paratesto. Quindici anni fa l'Unità uscì con, in allegato, i Vangeli. Pochi anni prima a dominare le vendite era stata La Lettera sulla Felicità di Epicuro, nell'edizione che Stampa Alternativa vendeva a mille lire. In entrambi i casi l'approccio sottinteso era eterodosso; in entrambi i casi struttura e sovrastruttura s'intrecciavano, il mezzo, per dirla con McLuhan, diventava contenuto.

In fondo, di tutta la macchina che chiamiamo editoria, il manoscritto non è che l'innesco, poco più di un pretesto. È dopo, grazie a chi ne cura l'editing o la distribuzione, a chi riesce a legarlo ad una moda, ad un evento, ad un ceto, a chi riesce ad imporlo in una vetrina, che quelle pagine vanno nelle classifiche invece che al macero.

Considerare un libro nel suo complesso, allora, può aiutarci a individuare quello "specifico femminile" che ha riempito tante biblioteche di critica letteraria e nessuna di marketing.

\section{L'ASCESA DELLE DONNE NEI GIORNALI E NEI LIBRI}

Solo negli anni '70 la redazione del Messaggero contava centotrentanove giornalisti e una giornalista. Oggi, nell'albo professionale le quote vanno parificandosi. Milly Buonanno, in Visibilità senza potere, ha ripercorso l'affermazione delle donne nell' ambito dell'informazione. Dal 1978, in pochi anni, con la proliferazione di canali televisivi, il totale delle giornaliste nel mondodell'informazione si moltiplicò per nove. Venticinque anni dopo scoppia la prima guerra del Golfo. È estate e molti giornalisti sono in ferie. Nelle redazioni sono rimasti i praticanti, i più giovani. Sono in gran parte donne e con i loro "pezzi" dal fronte riescono ad imporsi. Superando decenni di confino nelle sezioni della moda ma anche quella frivolezza che Miriam Mafai aveva attribuito loro in un convegno del '93. Poco dopo Ilaria Alpi, in Somalia per indagare su un traffico di armi e rifiuti tossici, veniva uccisa. Nel 1999, in Jugoslava, toccava a Maria Grazia Cutuli, del Corriere della Sera. Nel 1997 Milena Gabbanelli aveva iniziato la conduzione del suo programma Report, modello di giornalismo investigativo. Nel 2001, per un articolo sull'attentato delle torri gemelle, Oriana Fallaci si vide recapitare un mandato di cattura. Nel 2004 Dietlinde Gruber, volto del telegiornale, alle elezioni europee del 2004 conquista più di un milione di preferenze. Dal 2009 Bianca Berlinguer è la direttrice del telegiornale della terza rete nazionale, quello con il maggior tasso di giornaliste. Sotto la sua direzione arriva ad essere il più seguito. Tra il 2009 ed il 2011
Concita De Gregorio dirige l'Unità; negli stessi anni Norma Rangeri il Manifesto. Una delle sue giornaliste, Giuliana Sgrena, nel 2005 fu tenuta in ostaggio in Iraq.

Sempre più spesso le donne accedono ad incarichi direttivi, alle prime pagine, ai temi scottanti. Questo non ne ha tacitato lo spirito critico. Nel 2012 è nata Gi.u.li.a, rete di Giornaliste Unite Libere Autonome. Ciò per cui si batte è, "un cambiamento anche nella vita delle redazioni, piene di giornaliste ma ancora dominate da logiche einteressi maschili".

\section{COME VANNO, INVECE, LE COSE NELL'EDITORIA LIBRARIA?}

Cominciamo col dire che le donne che lavorano nella carta stampata sono tutte in debito colle pioniere degli anni ‘ 60 . Coi loro volantini, coi loro manifesti, coiloro periodici in realtà non di rado saltuari. Un tirocinio che offrì a tante ragazze l'occasione di dibattere, sperimentare edivulgare le proprie idee che magari poi travasarono nell' editoria libraria. Nel 1975 nasce la rivista DWF della Libreria delle donne di Milano e, pressocchè contemporaneamente, le case editrici: La Rosa, a Torino, la Tartaruga a Milano, Le Edizioni delle Donne a Roma. Mentre nelle redazioni dei giornali il peso delle donne è cresciuto nei primi anni '90, in quelle dei libri il fenomeno ha atteso qualche anno: è solo tra il 1998 ed il 2003 le case editrici aumentarono del 43\%.

Declino delle iniziative editoriali delle donne

Oggi l'intero settore pare declinare. Il sito della DWF non viene aggiornato da più di un anno. NoiDonne, un tempo legato al Partito comunista, ha saputo resistere alla clandestinità ma non al mercato: anni fa ha interrotto le pubblicazioni. Le ha riprese dopo qualche anno ma oggi a dirigerlo è Tiziana Bertolini, estranea per biografia, oltre che per questioni di anagrafe, ai dibattiti femministi di un tempo. Delle tre case editrici menzionate, due sono cessate ed una, La Tartaruga, langue come collana della Baldini Castoldi Dalai. Luciana Tufani, che avendo fondato nel 1996 la casa editrice omonima e già nel 1980 la rivista Leggere Donna, conferma il legame tra stampa periodica e libraria, lamenta una caduta degli abbonamenti ed è ridotta a produrre un libro all'anno. TutteStorie, rivista di racconti al femminile nata nel 1990, ha chiuso i battenti ormai da un decennio.

Nel 1991, nel convegno In forma di rivista, Anna Maria Crispino enumerava i punti dolenti dell'editoria delle donne: costi elevati, tirature ridotte, penuria di mezzi per promuoversi. La distribuzione ne risultava soffocata e tutto il settore relegatoin un circuito chiuso. A prima vista, oggi la situazione non sembrerebbe cambiata. All'inserto Tuttolibri della Stampa che già il 7 marzo 1992 si chiedeva cosa rimanesse della stampa femminista, a prima vista verrebbe da citare solo qualche bollettino per reduci sessantottine. 
Nel 2001, il supplemento al n. 58/59 della rivista Quaderni di via Dogana s'intitolava Un'eredità senza testamento. Tra le riflessioni contenute nel volume spicca quella di Luisa Muraro: “Il femminismo non è diventato qualcosa di memorabilein senso forte: qualcosa che si fa ricordare perchè iscritta fra le cose irrinunciabili." Per Ida Dominjanni il disinteresse quando non il rifiuto verso il femminismo da parte delle giovani generazioni nasce da un equivoco: "noi abbiamo criticato il fallologo centrismo,e il senso comune ci registra come donne falliche." Recitare un de profundis sarebbe però fuori luogo.

Indizi di controtendenza

Così chiudeva Luciana Tufani un articolo intitolato Bilanci su un numero del 2009 di Leggere donna. "Ha un po' l'aria del testamento e in parte lo è, anche se, pur sapendo di essere mortale, non riesco e non voglio convincermi di esserlo. Per ora, comunque, non ho intenzione di morire: non ho tempo."

Numerose sono le pubblicazione che resistono e si rinnovano. Leggendaria, nato nel 1997, sotto la direzione di Anna Maria Crispino ha di recente affrontato un taglio dei fondi senza rinunciare a gettare il proprio sguardo ben oltre la semplice rassegna di recensioni. Luciana Percovich cura Le Civette, collana che la casa editrice Venexia dedica alla riscoperta del femminile nel sacro. La più nota Giunti ormai da un quarto di secolo ospita la collana Astrea che dà spazio alle immigrate. Non mancano le novità. Una casa editrice giovane è Il caso e il vento. Attente all'oralità, le socie promuovono iloro prodotti alla maniera di Fahrenhei 451, organizzando serate in cui "persone libro" 1 declamano a memoria. Meno ideologica l'esperienza della Caravan, cooperativadi quattro ragazze che hanno eletto come sede la casa internazionale delle donne di Roma. Ci hanno riferito di come, in quanto donne, talvolta stentino ad ottenere dal tipografo che le consegne degli ordini siano puntuali.

Tanta parte dei fermenti editoriali delle donne ormai da tempo aggirano tuttavia questo tipo di contrattempi. Sono le decine di siti internet, da quelli istituzionali ai blog in cui associazioni, organi o anche singoli individui discutonoo anche solo raccontano. Eredi, quanto a povertà di mezzi, a vivacità di idee, talvolta a radicalità delle proposte, delle pubblicazioni degli anni '60 e '70.Sono loro che possono in qualche modo assolvere alla funzione che avevano Fuori!, del Vaso di Pandora, Effe e tante altre iniziative cessate ormai da tempo. Parliamo di siti come www.sorelleditalia.net, www.ellexelle.com, vasettodipandora.wordpress. com/cucu/ www.womanjournal.it/, http://www.levocianti.it/. Vengono, del resto, dal mondo dei blog due dei maggiori successi degli ultimi anni: La ballata delle prugne secche, di Pulsatilla, e Il mondo deve sapere, di Michela Murgia. Tra tutti spicca, per visibilità e, come si dice in gergo, "contatti", la 27 ora, il blog del Corriere della Sera.
E qui arriviamo al terzo indizio della vitalità dell'editoria delle donne: la penetrazione del proprio messaggio. I concetti che qualche decennio fa circolavano tra poche pioniere, oggi, come ci ha fatto notare Piera Codognotto in un incontro a Firenze presso la biblioteca dove lavora, hanno attecchito, si sono radicate. Pensiamo Filema e Liguori, case editrici a stretto contatto con l'università di Napoli. Ma un ripensamento dei ruoli di genere ha preso piede ormai anche nella cultura popolare. Se si legge la posta del cuore dei rotocalchi di qualche decennio fa si rimane colpiti da quanto le risposte esortassero alla conservazione dei ruoli tradizionali. Oggi, invece, alcuni di quei settimanali e mensili denunciano i casi di violenza di genere. In diverse case editrici anche non programmaticamente femministe, la pratica dei rapporti orizzontali è diventata quotidiana. È il caso di Iperborea, Voland, Zandonai, case di donne che, peraltro, rivolgendosi nel solco di Fernanda Pivano a letterature come la scandinava e la slava, confermano le teorie sulla vocazione della donna a tradurre, sulla sua abitudine storica a maneggiare il semantico per esprimere il semiotico. È il caso anche di Biancoenero che con caratteri e impaginazione appositi apre le proprie pagine ai dislessici. Ma è anche il caso di tante case che declinano nell'editoria la vocazione alla cura delle donne, come Orecchio Acerbo e Manidistrega.

Non mancano le sorprese. Una donna dirige Reonir comic, casa di fumetti, e di donne sono, a detta degli editori che abbiamo consultato, la maggior parte dei manoscritti erotici che arrivano nelle redazioni. Se le gialliste italiane rimangono rare, è la casa che fu di Elvira Sellerio ad esportare il commissario Montalbano. A prima vista pare invece latitare, tra le case editrici di donne, un marchio che si interessi di politica. A ben guardare, invece, più che alla teoria, della politica alle donne pare importare la prassi. Terre di mezzo è partita con un giornale di strada diffuso da extracomunitari, ha introdotto in Italia il concetto di decrescita, anni fa ha prodotto un gioco di società concepito dai reclusi di San Vittore e da anni organizza la fiera $\mathrm{Fa} l a$ cosa giusta!, vero evento dello sviluppo sostenibile.

Peso (numerico e non) delle donne in editoria

Se proponete un testo ad un editore ci sono ottime possibilità che a leggerlo, correggerlo, recensirlo, promuoverlo, siano donne. Sono tante, insomma, le case editrici di donne, e tantissime le donne occupate in editoria. Logico riflesso dello scarto tra lettrici e lettori che del 1988 si è triplicato, raggiungendo i diciotto punti percentuali. Tra i librai la percentuale di donne è oltre il 70\%. Quattro libri su dieci sono scritti da donne, ma solo uno dei tre più venduti nell'ultimo anno era scritto da un uomo. E quanto al legame che c'è tra lettrici ed editrici, basti pensare che Laura Lepetit dichiarò di aver fondata la Tartaruga con lo scopo di leggere opere che in Italia non aveva occasione di trovare. Ormai da diversi anni Pappalardo, nella sua Guida agli editori che ti pubblicano, offre una puntuale rendicontazione di chi ricopre i principali incarichi 
nelle case editrici italiane, che trova un puntuale riscontro nei dati dell'Associazione Italiana Editori: su tre persone che ogni anno accedono ad una professione legata all'editoria, due sono donne. È un dato che ricorda quelli dei concorsi per diventare giornalisti. Per dare un'idea del contesto in cui tutto ciò avviene, ricordiamo che secondo il Global Center Gap Report, l'Italia è al novantaseiesimo posto al mondo per quanto riguarda la partecipazione delle donne all'economia.

Il riconoscimento delle capacità delle donne, in editoria, è unanime. In misura perfino maggiore del banale riscontro che potrebbe dare una lettura degli organigrammi aziendali. Mentre è solo dal 12 agosto, e per legge, che i Consigli d'amministrazione delle società pubbliche e di quelle quotate in borsa devono essere composte almeno per un terzo da donne, i dati dell' AIE registrano che nel aziende editoriali la percentuale di donne che coprono incarichi direttivi è del 40,6, addirittura $49 \%$ nella piccola editoria; era del 27\% nel 1991.

Il merito delle editrici

Dedicare, come nel 1996, un'edizione della Fiera del libro di Torino ai rapporti tra donne ed editoria oggi sarebbe impensabile. Nel mondo dei libri le donne hanno smesso di essere un'eccezione. Il "merito delle donne", in editoria, è quasi scontato. Susanna Romano, fondatrice dell'agenzia letteraria Menabò, in un incontro presso una libreria di Trastevere diha riferito che si riconosce loro una particolare attenzione, precisione, solerzia. "per fabbricare un libro ci vogliono cure e pensieri come per un figlio -ha dichiarato Laura Lepetit in un'intervista- bisogna inventarlo, preparalo, seguitlo fino a che esce di casa e poi trepidanti seguire i suoi successi o insuccessi: un lavoro perfetto per una donna".

Se non siedono ancora più di frequente nelle stanze dei bottoni, per Alberto Castelvecchi, un tempo editore oggi esperto d'immagine, è perché spesso le donne preferiscono operare dove davvero si decide la politica editoriale, sugli sgabelli delle redazioni più che nelle poltrone delle direzioni. Anni fa il compianto Gianfranco Cosma fondatore della Palomar, per indicarci l'acume delle proprie e altrui collaboratrici ci accompagnò sottobraccio tra gli stand della fiera di Roma, dove donne illustravano, vendevano, impacchettavano, organizzavano. Nella stessa occasione chiedemmo a Marco Cassini, fondatore della Minimum fax, perchè avesse lasciato le redini della sua creatura a Martina Testa; “è più brava di me”, ci rispose.

Per qualcuno che tuttora fa notare il numero sospetto, tra gli addetti stampa, di ragazze messe quasi in vetrina per accaparrarsi recensioni, c'è la fondatrice di Edizioni Clandestine che rievoca come qualcosa di remoto l'epoca in cui doveva affrontare venti distributori uomini. Louise Read, fondatrice della Socrates, cosmopolita per formazione, frequentazioni e produzione, e quindi col metro di paragone di realtà che spesso sottolineano le arretratezze italiche, ha negato di essersi imbattuta, nella sua ventennale attività, in qualsiasi forma di sessismo.

E-book, editrici?

Dalla direzione della casa editrice che da sola vale un terzo del fatturato del settore al fervore delle stagiste, insomma, l'editoria, in Italia, è sempre più campo d'espressione delle donne. Paventeremmo la possibilità di una loro egemonia culturale prossima ventura se le nuove forme di fruizione che ci offre la tecnologia non mettessero in discussione lo stesso concetto di pubblicazione. Ora che la sensibilità della donna scorre tra le rotative come una nuova feconda cellulosa, ecco che le parole diventano chip. A seconda dell'evoluzione del mercato essere presenti nell'editoria potrà essere per le donne un'opportunità o la conquista di un guscio vuoto. Cosa troveremo di femminile in una casa editrice quando i libri, invece di sfogliarli, bisognerà scaricarli? Gli e-book favoriranno gli oligopoli o il self publishing? Possiamo provare a rispondere. Partendo dall'osservazione che, oltre ad un metodo di lavoro e agli approdi teorici dei dibattiti, ciò che le pioniere degli anni ' 60 hanno trasmesso alle addette ai lavori di oggi è un'apertura all'innovazione. Se l'Associazione Italiana Editori ha scelto Cristina Mussinelli come responsabile per le nuove tecnologie, in altre parole, èforse, indirettamente, anche perchè già nel 1970 Rosi Braidotti si occupava di Cyber femminismo. E non è un caso se oggi, sul blog che tiene sul Fatto quotidiano, sia una donna, Margherita Loy a porsi delle domande sull'eventuale discriminazione insita inun sistema che peremtte di accedeere ai libri solo ai possessori di carta di credito. Mentre sul sito della Socrates si legge, senza troppe indecisioni: “siamo entusiasti degli e-book". Forse perchè, anche per motivi ideologici, le donne che negli anni'60 cercavano nuove forme di distribuzione non ebbero mai, col mercato, un rapporto passivo.

L'eredità delle pionere

Nel 2005 Andrè Shiffrin denunciò i pericoli degli oligopoli e delle logiche industriali in editoria. Ad andare oltre, a dimostrare che un'altra editoria è possibile sono state soprattutto le donne. Farlo attraverso il catalogo, però, non basta. Molte sono le iniziative che lo traducono in atti concreti: l'adozione di prodotti ecologici,la ricerca di canali alternativi di finanziamento e distribuzione, la proposta alle dipendenti di condizioni non vessatorie, il rifiuto dell'editoria a pagamento. C'è, inoltre, un'altra eredità che il femminismo, ci pare, ha lasciato alle donne che si muovono nell'editoria di oggi. Consiste nel respingere la consuetudine, mettere in discussione il passato, rifiutare il senso comune. Si può cadere nell'errore di identificare la scrittura femminile con Liala ma sulle case editrici non ci sono stereotipi.Se mancano le scrittrici comiche ad esempio, è proprio a quel settore che la più giovane editrice d'Italia, Marianna Martino, dedica la sua ditta. La Zandegù, è vero, 
è fallita, ma invitiamo tutti a leggere il commiato che la fondatrice ha lasciato sul sito in cui ringrazia sé stessa per "le idee, l'incoscienza e le sette camicie che ho sudato".

Colpisce, specie nelle prime imprese femministe, la concretezza. Da dilettanti qualche decennio fa, anteponevano il fine ai mezzi, lo scopo all'organizzazione. Le cronache dell'epoca resocontano di redazioni gremite e confuse, di dibattiti che non tenevano conto dei ritmi del mercato, di scelte editoriali che ignoravano i vincoli di bilancio. Dagli errori di una generazione, le editrici hanno tratto una lezione. Oltre a quella ideologica, essa consiste nel non dimenticare il motivo per cui si è nati. Perseguirlo, comporta sopravvivere, e viceversa. Perciò le editrici curano i conti. Consapevolmente o no, il destino di testate sorde alle raccomandazioni de ragioniere, ha fatto scuola. Da ciò discende una serie di differenze dai ciclostilidi qualche decennio fa. L'aspetto formale, intanto. Le case editrici di donne lo tengono in considerazione. Spesso spiccano la consistenza della carta, la cura delle illustrazioni, la precisione della confezione. A volte aumenta l'appetibilità del prodotto, a volte sono perfettamente strumentali. Ad esempio molte case propongonoprodotti per l'infanzia, sui quali le illustrazioni sono fondamentali. Nottetempo, fondata da Ginevra Bompiani e Roberta Einaudi, deve il proprio nome al rivolgersia quei lettori che sfogliano qualche pagina a letto, prima di assopirsi. Il formato dei suoi prodotti è pertanto maneggevole e la dimensione dei caratteri caratteristica. Torniamo, quindi, al punto di partenza, al peritesto: cioè al volume. Anche attraversodi esso, con la fusione tra etica ed estetica, delle femministe di un tempo le case di oggimantengono un carattere sostanziale: il senso di una missione.

\section{RIFERIMENTI BIBLIOGRAFICI}

AA.VV. Convegno "Mediawomen. Etica e potere nel giornalismo delle donne" atti originariamente pubblicati in Problemi dell'informazione n. 3, 1993, poi come Guardare con gli occhi in Visibilità senza potere, Firenze, maggio 1993.

Bochicchio, G.-De Longis, R., La stampa periodica femminile in Italia, Milano, Biblink, 2009.

Buonanno, M., Visibilità senza potere, Liguori, Napoli, 2005.

Codognotto, P., Editoria femminista in Italia, Roma, Associazione librerie italiane, 1997.

Dominjanni, I., La politica nelle riviste delle donne, Bib. d. d. di P., Parma, 1995.

Franchini, S.,-Soldati, S., Donne e giornalismo: percorsi e presenze di una storia di genere, Franco Angeli, Milano, 2005.

Palumbo, V., L'era delle ragazze alfa,Fermenti, Roma, 2009.

Pappalardo, L., La guida agli editori che ti pubblicano, Delos Books, Milano, 2011.

Pezzuoli, G., La stampa femminile come ideologia, Il formichiere, Milano, 1975.
Pulsatilla, La ballata delle prugne secche, Castelvecchi, Roma, 2006.

Ragone, G., L'editoria in Italia, Liguori editore, Napoli, 2005.

Schriffin, A., Editoria senza editori, Bollati Boringhieri, Milano, 2004

Tatafiore, R., 100 titoli, Tufani, Ferrara, 2003.

Tufani, L., Leggere donna, guida all'acquisto, Tufani, Milano, 1996

Turi, G., Storia dell'editoria nell'Italia contemporanea, Giunti, Milano, 1997.

Vanuuncchi, I F., Introduzione allo studio dell'editoria, ed. Bibliografica, Milano, 2004.

Articoli

Muraro, A., “Un'eredità senza testamento", in Quaderni di via Dogana, n. 58/59, Milano, 2001.

Rusconi, M., "Tre editrici e un'unica passione", in Tuttestorie, n.4, 1995.

Ventavoli, B., "Donne di carta. Che cosa e' rimasto dell' editoria femminista", in Tuttolibri, inserto de "La Stampa", 7 marzo 1992.

Vittori, M.V., "Editoria declinata al femminile, una piccola grande storia", in Liberazione, 30 maggio 2005.

\section{Sitografia}

http://www.aie.it/

http://giulia.globalist.it/Detail_SimplePage_Results?ID=127\&loid=111

http://www.sorelleditalia.net

http://www.ellexelle.com

http://vasettodipandora.wordpress.com/cucu/

htpp://www.womanjournal.it/

http://www.levocianti.it/

http://pulsatilla.org

http://www.dwf.it

http://www.ilcasoeilvento.it

http://tropicodellibro.it/notizie/donne-lavoratrici

http://donneculturaeinformazione.blogspot.it/2010/04/editoria-declinata-al-femminile-una

$\mathrm{htm}$

http://27esimaora.corriere.it

http://www.ilfattoquotidiano.it/2012/08/11/323857/323857/

http://www.editorisardi.it/index.php?option=com_content\&view=article\&id=171:editoria-alfemminile\&catid $=58$ :press\&Itemid $=74$

http://www.elle.it/Magazine/Nuova-casa-editrice-ghena-francesca-gagliardi

http://www.edizionisocrates.com/ 
http://www.corriere.it/cronache/08_dicembre_21/editoria_potere_rosa_cristina_ taglietti_55b80cba-cf3b-11dd-9e84-00144f02aabc.shtml

http://www.ilmenabo.it/lsc_programma.php

http://donneculturaeinformazione.blogspot.it/2010/04/editoria-femminilefemminista.html

h t t p:// w w w. m clink.it/n/d w press/libro/libro $15 . \mathrm{ht}$ m http://www.enciclopediadelledonne.it/index.php?azione=pagina\&id $=460$ 